\title{
Distribución geográfica de los ortópteros (Insecta: Orthoptera) presentes en las provincias biogeográficas de Atacama y Coquimbo (Chile) ${ }^{1}$
}

\author{
Fermín M. Alfaro², Jaime Pizarro-Araya ${ }^{3}$, Luis Letelier ${ }^{4}$ \\ y Jorge Cepeda-Pizarro ${ }^{5}$
}

\begin{abstract}
RESUMEN
Mediante la revisión de material de referencia, literatura y prospecciones entomológicas se documentó la composición taxonómica del orden Orthoptera para las provincias biogeográficas de Atacama y Coquimbo y se estableció la distribución espacial de sus especies en relación a las formaciones vegetales descritas para dichas provincias. Se registró la presencia de 68 especies, 37 géneros y 9 familias. Tettigoniidae fue la familia más diversa, seguida de Proscopiidae y Acrididae. La mayor riqueza específica se observó solo en cuatro de las 23 formaciones vegetales analizadas. El mayor número de especies se registró en el matorral estepario costero (47 especies), seguido del matorral estepario interior (28 especies), estepa arbustiva de la precordillera de Coquimbo (14 especies) y desierto costero del Huasco (13 especies). El presente catastro georreferenciado constituye una primera aproximación de los patrones distribucionales de las especies de Orthoptera pertenecientes a estas áreas biogeográficas.
\end{abstract}

Palabras clave: Orthoptera, Chile, diversidad, distribución, desierto costero.

\begin{abstract}
By means of reference material and literature reviews, as well as entomological surveys, we documented the taxonomic composition of the order Orthoptera within the biogeographic provinces of Atacama and Coquimbo, and identified the relationships between the spatial distribution of species and the plant formations described from these provinces. We recorded the presence of 68 species, 37 genera, and 9 families. The most diverse family was Tettigoniidae, followed by Proscopiidae and Acrididae. High species richness was observed in only 4 of the 23 plant formations analyzed. The areas with the highest number of species were
\end{abstract}

\footnotetext{
1 Los autores agradecen a Iván Benoit (Corporación Nacional Forestal del Gobierno de Chile, CONAF) por la ayuda en los permisos y facilidades para trabajar en las áreas del SNASPE. A los curadores de las colecciones nacionales y extranjeras por la ayuda entregada. Fermín M. Alfaro agradece a la beca CONICYT-PCHA/Magíster Nacional/2013-22130123. Luis Letelier agradece a CONICYT por la beca de postgrado dentro del programa Becas Chile en el Extranjero. Este trabajo fue financiado a través de los proyectos DIULS-PR13121, VACDDI001 (J.P.A.) y CDI12 (J.C.P.) de la Universidad de La Serena (Chile). Artículo recibido el 24 de abril de 2012, acepta-
}

do el 30 de septiembre de 2012 y corregido el 18 de diciembre de 2012 .

2 Laboratorio de Genética y Evolución, Departamento de Ciencias Ecológicas, Universidad de Chile (Chile). E-mail: fermin.alfaro@ug.uchile.cl

3 Laboratorio de Entomología Ecológica, Departamento de Biología, Universidad de La Serena (Chile). E-mail: japizarro@userena.cl

4 Centro de Investigaciones en Ecosistemas, Universidad Nacional Autónoma de México (México). E-mail: Iletelier@cieco.unam.mx

5 Laboratorio de Entomología Ecológica, Departamento de Biología, Universidad de La Serena (Chile). E-mail: jcepeda@userena.cl 


\begin{abstract}
the coastal shrubby steppe (47 species), the interior shrubby steppe (28 species), the shrubby steppe of the precordillera of Coquimbo (14 species), and the coastal desert of Huasco (13 species). This georeferenced survey is a first attempt to identify the distribution patterns of orthopteran species within these biogeographic areas.
\end{abstract}

Key words: Orthoptera, Chile, diversity, distribution, coastal desert.

Las provincias biogeográficas de Atacama $\left(18-28^{\circ} \mathrm{S}\right)$ y Coquimbo $\left(28-32^{\circ} \mathrm{S}\right)$ (Morrone, 2006) poseen dentro de su extensión la franja meridional del desierto costero transicional de Chile, área que representa el límite septentrional del foco de diversidad vegetal hotspots para Chile Central (Cowling et al., 1996; Gastón, 2000), y que posee una artropodofauna particular en términos de riqueza específica (Cepeda-Pizarro et al., 2005a; Cepeda-Pizarro et al., 2005b; Pizarro-Araya et al., 2008; Alfaro et al., 2009; Pizarro-Araya et al., 2012a; Alcayaga et al., 2013), endemismos (Jerez, 2000; Pizarro-Araya y Jerez, 2004; Pizarro-Araya et al., 2012b) y distribuciones restringidas (Pizarro-Araya \& Flores 2004; Alfaro et al., 2009).

El conocimiento de los artrópodos para estas regiones se basa en estudios taxonómicos y sistemáticos, siendo la ecología y biogeografía temáticas poco abordadas para esta zona del norte de Chile (Pizarro-Araya et al., 2008). Dentro de la artropodofauna, la mayoría de los trabajos son de carácter taxonómicos y sistemáticos de insectos, en particular Coleoptera (Jerez, 2000; Pizarro-Araya et al., 2008; Flores \& Pizarro-Araya, 2012) y de arácnidos, con énfasis en Scorpiones (Ojanguren-Affilastro, 2002; Ojanguren-Affilastro, 2004; OjangurenAffilastro, 2005; Ojanguren-Affilastro, 2006; Ojanguren-Affilastro et al., 2007; OjangurenAffilastro et al., 2012), Araneae (Grismado, 2010; Lucas \& Indicatti, 2010) y Solifugae (Valdivia et al., 2008; Valdivia et al., 2011).

En Chile, el orden Orthoptera, está representado por 149 especies agrupadas en 69 géneros y 13 familias (Elgueta et al., 1999; Vera, 2006) las que se distribuyen dentro de la totalidad del territorio continental, desde el nivel del mar hasta más de 4.000 metros de altitud (Elgueta et al., 1999). El nivel de endemismo de Orthoptera en Chile representa el 56\% de los géneros y el $75 \%$ de las especies (Vera, 2006). El actual conocimiento del orden Orthoptera, se basa en un catálogo a nivel nacional de las especies (Elgueta et al., 1999), estudios morfométricos y de irrupciones poblacionales de Elasmoderus wagenknechti (Liebermann) (Tristiridae) para el semiárido de Chile (Moroni, 1972; Cepeda-Pizarro et al., 2003; Cepeda-Pizarro et al., 2006; Cepeda-Pizarro et al., 2007) y de riqueza específica en sectores de secano y cultivos en la cuenca del Elqui (Región de Coquimbo) (Pizarro-Araya et al., 2009; Alfaro et al., 2011).

Considerando que no existen antecedentes actualizados sobre la distribución de las especies de Orthoptera para las provincias biogeográficas descritas anteriormente y la necesidad de generar catastros que sirvan como información base para identificar posteriormente áreas de endemismos, los objetivos del presente trabajo fueron 1) documentar la composición taxonómica del orden Orthoptera para las provincias biogeográficas de Atacama y Coquimbo y 2) establecer la distribución espacial de sus especies para dichas provincias.

\section{Materiales y métodos}

Área de estudio

La distribución de las especies de Orthoptera consideró como área de estudio las provincias biogeográficas de Atacama y Coquimbo, siguiendo lo propuesto por Morrone (2006).

La provincia biogeográfica de Atacama comprende el norte de Chile entre los $18^{\circ} \mathrm{y}$ $28^{\circ}$ de latitud sur y forma parte de la zona de transición Sudamericana (Morrone, 2004a; Morrone, 2004b; Morrone, 2006). De acuerdo a Gajardo (1994) la vegetación de esta zona geográfica ha sido definida como per- 
teneciente a la Región del Desierto, con las subregiones del Desierto Absoluto, Desierto Costero y Desierto Florido. Las precipitaciones en el Desierto Absoluto son escasas al igual que la vegetación, la cual está representada por comunidades de Atriplex deserticola Phil., Lycium minutifolium J. Remy, Nolana baccata (Lindl.) Dunal y Cryptantha parviflora (Phil.) Reiche. El Desierto Costero ubicado más al sur entre los $25^{\circ}$ y $32^{\circ}$ de latitud sur posee un mayor aporte de precipitaciones producto de la neblinas costeras, lo que permite el desarrollo de una mayor vegetación representada por comunidades de Cassia brongniartii (Gaudich.) H.S. Irwin \& Barneby, Dinemandra ericoides A. H. L. Juss., Mathewsia incana Phil. y por varias especies del género Nolana. El Desierto Florido, perteneciente a la sección más meridional, recibe precipitaciones periódicas lo que permite el desarrollo de especies efímeras y anuales. Las comunidades características están representadas por Skytanthus acutus Meyen, Rhodophiala ananuca (Phil.) Traub, Encelia canescens Lam., varias especies del género Nolana y Balsamocarpon brevifolium Clos (Gajardo, 1994). Para el tramo comprendido entre los $21^{\circ} 28^{\prime} \mathrm{S}$ y $25^{\circ} 40^{\prime} \mathrm{S}$, y perteneciente a la región de Antofagasta, Squeo et al. (1998) han determinado que la mayor diversidad de la flora vascular se presenta en las formaciones vegetales costeras, destacando el Desierto Costero de Taltal como el área con mayor diversidad. Por otro parte, Squeo et al. (2008) han registrado para la franja comprendida entre los $26^{\circ}$ y $29^{\circ}$ de latitud Sur la presencia de 1.099 especies de plantas vasculares, de las cuales un $54,3 \%$ son endémicas para Chile.

La provincia biogeográfica de Coquimbo forma parte de la subregión chilena central en la región Andina y comprende el Centro Norte de Chile desde los $28^{\circ}$ a los $32^{\circ}$ de latitud sur (Morrone, 2000; Morrone, 2002; Morrone, 2004b; Morrone, 2006). La vegetación del área forma parte de la Región del Matorral y del Bosque Esclerófilo y de la subregión del Matorral Estepario. Esta zona se caracteriza por la presencia de precipitaciones irregulares y bajas. Las comunidades características están representadas por Adesmia microphylla Hook. et Arn., Senna cumingii (Hook. \& Arn.) H.S.Irwin \& Barneby, Lithrea caustica (Molina) Hook. et Arn., Colliguaja odorifera Molina, Helenium aromaticum (Hook.) L.H.Bailey,
Sagina apetala Ard., Peumus boldus Molina y Podanthus mitiqui Lindl. (Gajardo, 1994). De acuerdo a Squeo et al. (2001), la franja latitudinal entre $\operatorname{los} 29^{\circ} 00^{\prime} \mathrm{S}$ y $32^{\circ} 10^{\prime} \mathrm{S}$, perteneciente a la región de Coquimbo, registra un total de 1.478 especies nativas de las cuales el $53,5 \%$ de ellas son endémicas de Chile.

\section{Distribución geográfica}

Se realizó una recopilación de registros distribucionales de Orthoptera a partir de la revisión de material de referencia conservado en las colecciones entomológicas del Museo de Zoología de la Universidad de Concepción (MZUC), Museo Nacional de Historia Natural, Santiago (MNHN), Instituto de Entomología, Universidad Metropolitana de Ciencias de la Educación, Santiago (IEUMCE), Colección Entomológica de la Universidad de Tarapacá, Arica (IDEA), Laboratorio de Entomología Ecológica del Departamento de Biología de la Universidad de La Serena, La Serena (LEULS), Museo Argentino de Ciencias Naturales Bernardino Rivadavia, Argentina (MACN-Ar), y colección particular de Juan Enrique Barriga (JEBC). Los registros de colección fueron complementados con datos distribucionales señalados en literatura (i.e. Cigliano et al., 1989; Elgueta et al., 1999), en páginas web (Eades et al., 2013), capturas con trampas de caída (pitfalls traps) y colectas manuales realizadas entre 2004 y 2011. Todas las localidades de muestreo fueron referenciadas geográficamente mediante GPS (Etrex-Personal Navigator, Garmin). El material recolectado se identificó a nivel específico mediante literatura especializada (i.e. Mello-Leitão, 1939; Ronderos, 1972; Cigliano et al., 1989; Artigas, 1994) y comparación con material del museo Nacional de Historia Natural (MNNC, Santiago, Chile) (Cuadros $\mathrm{N}^{\circ} 1$ y $\mathrm{N}^{\circ} 2$ ). El material colectado está depositado en la colección del Laboratorio de Entomología Ecológica de la Universidad de La Serena (LEULS), La Serena, Chile y en el Museo Nacional de Historia Natural, Santiago (MNHN).

\section{Formaciones vegetales y distribución espacial de Orthoptera}

La distribución espacial de Orthoptera se determinó a través de la presencia o ausencia 
de las especies en las formaciones vegetales descritas por Gajardo (1994) para las provincias biogeográficas de Atacama y Coquimbo.

Los mapas de distribución se generaron con la ayuda del software ArcGIS 9.3 a partir de los registros generados en el estudio y las bases de datos recopiladas. Se utilizó como base cartográfica, los límites regionales comprendidos dentro del área de estudio (escala 1:250.000) e imágenes de satélite del área de estudio. Toda la cartografía utilizada se georreferenció en UTM Datum WGS 84 huso 19S.

Para determinar las formaciones vegetales en las cuales se distribuyen las especies de Orthoptera, los registros fueron cruzados con la capa digital (archivo shape para ArcView) de las comunidades de Gajardo (1994) por medio de un "spatial join", en donde a cada registro se le asignó la formación vegetal correspondiente, posterior a esto, por medio de Crystal Reports 7.0 se elaboró una tabla cartesiana de resumen, cuyas entradas fueron los nombres de las formaciones vegetales de Gajardo (1994) y los géneros de Orthoptera. La presencia o ausencia de las especies en cada una de las formaciones vegetales y la fuente de información del registro se detallan en el Cuadro $\mathrm{N}^{\circ} 2$.

\section{Composición taxonómica de Orthoptera}

Se registró la presencia de 68 especies, 37 géneros y 9 familias. Tettigoniidae fue la familia más diversa (12 géneros, 24 especies), seguida de Proscopiidae (seis géneros, 14 especies) y Acrididae (seis géneros, 11 especies). Tettigoniidae representó el 35,3\% de la riqueza específica total observada en el sitio de estudio. Las familias Raphidophoridae, Romaleidae y Tetrigidae estuvieron representadas solo por un género y una especie (Cuadro $N^{\circ} 1$ ). La riqueza específica y genérica de Orthoptera registrada en este estudio representó el $45,5 \%$ y $53,7 \%$, respectivamente respecto del conocimiento de este taxón para Chile.

La presencia o ausencia de las especies de Orthoptera en las formaciones vegetales se entrega en el Cuadro $N^{\circ}$ 2. La mayor riqueza específica se observó solo en cuatro de las 23 formaciones vegetales analizadas. El mayor número de especies se registró en el Matorral estepario costero (MEC) con 47 especies, seguida del Matorral estepario interior (MEI) con 28 especies, Estepa arbustiva de la precordillera de Coquimbo (EAPC) con 14 especies y Desierto costero del Huasco (DCH) con 13 especies. Las restantes formaciones presentaron un menor número de especies (Figura $N^{\circ} 1$ ).

\section{Cuadro $\mathrm{N}^{\circ} 1$}

Composición taxonómica de Orthoptera, en términos de número de familias, géneros, especies y registros distribucionales en las provincias biogeográficas de Atacama y Coquimbo $\left(18^{\circ}-32^{\circ} \mathrm{LS}\right)$

\begin{tabular}{|c|c|c|c|}
\hline Familias & Géneros & Especies & Registros GPS \\
\hline Rhaphidophoridae & 1 & 1 & 1 \\
\hline Tettigoniidae & 12 & 24 & 61 \\
\hline Gryllidae & 3 & 3 & 35 \\
\hline Proscopiidae & 6 & 14 & 84 \\
\hline Tristiridae & 4 & 6 & 81 \\
\hline Ommexechidae & 3 & 7 & 34 \\
\hline Romaleidae & 1 & 1 & 1 \\
\hline Acrididae & 6 & 11 & 102 \\
\hline Tetrigidae & 1 & 1 & 4 \\
\hline Total & 37 & 68 & 403 \\
\hline
\end{tabular}

Fuente: Elaboración propia. 
PRESENTES EN LAS PROVINCIAS BIOGEOGRÁFICAS DE ATACAMA Y COQUIMBO (CHILE)

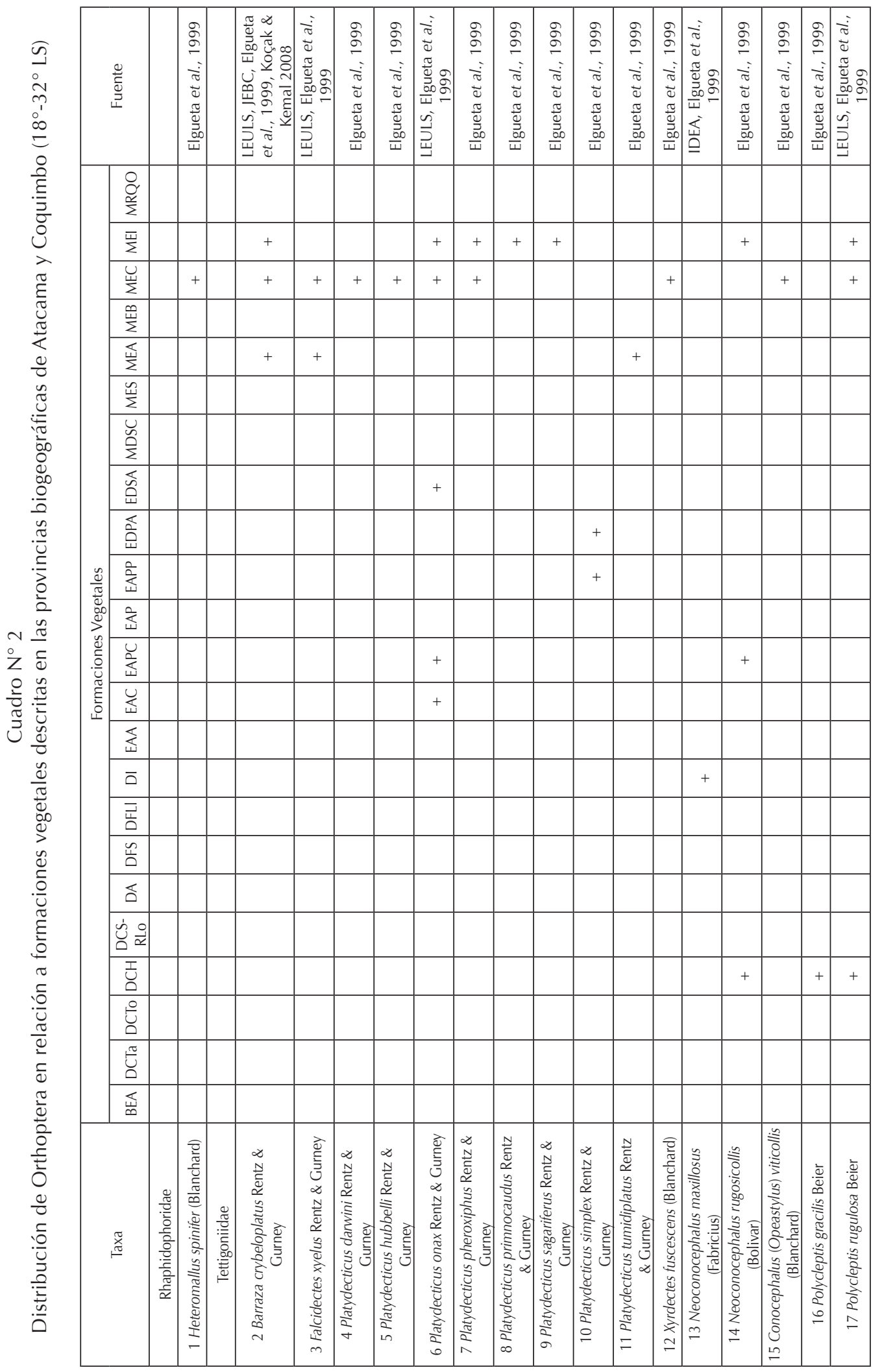




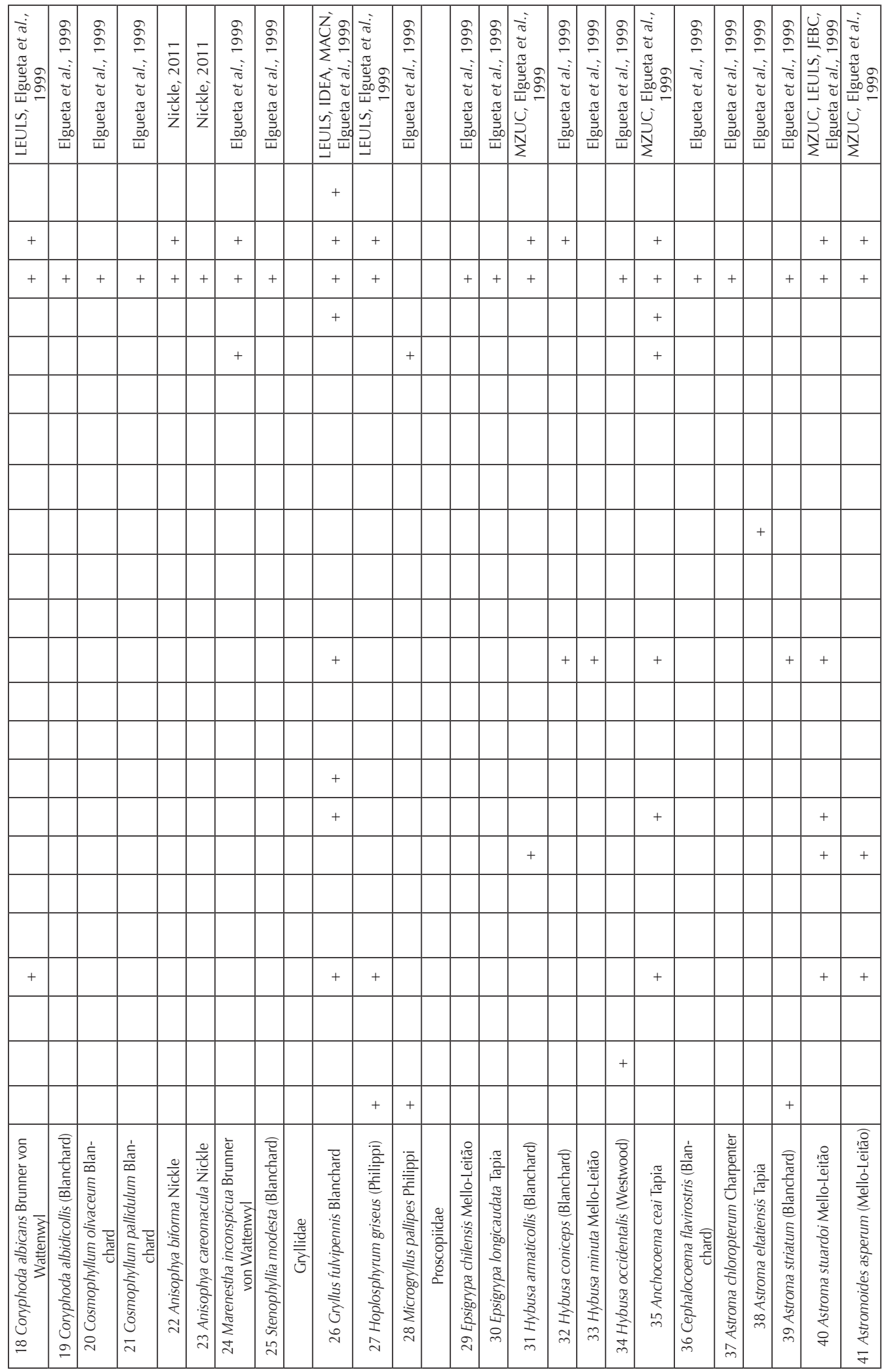




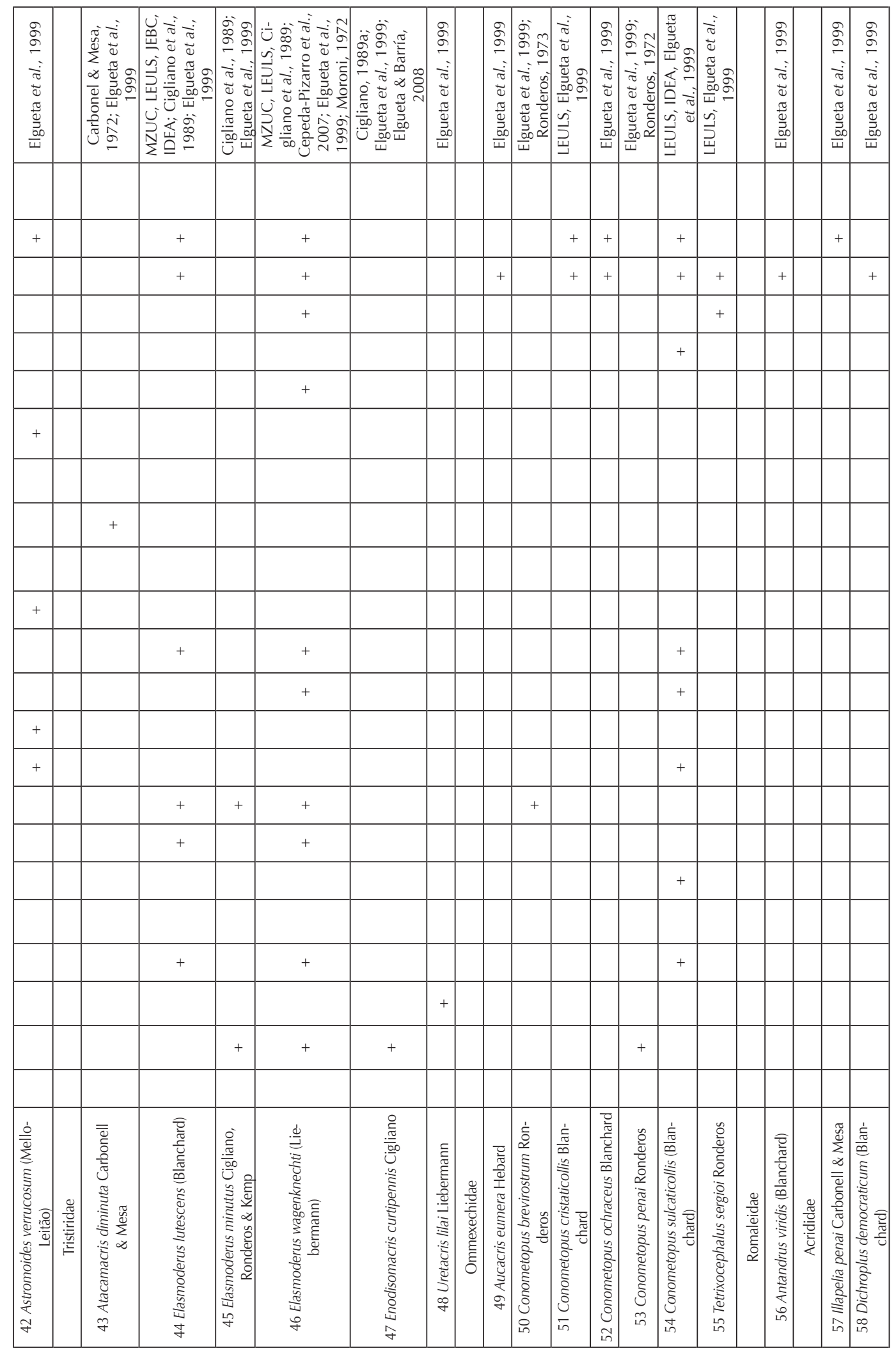




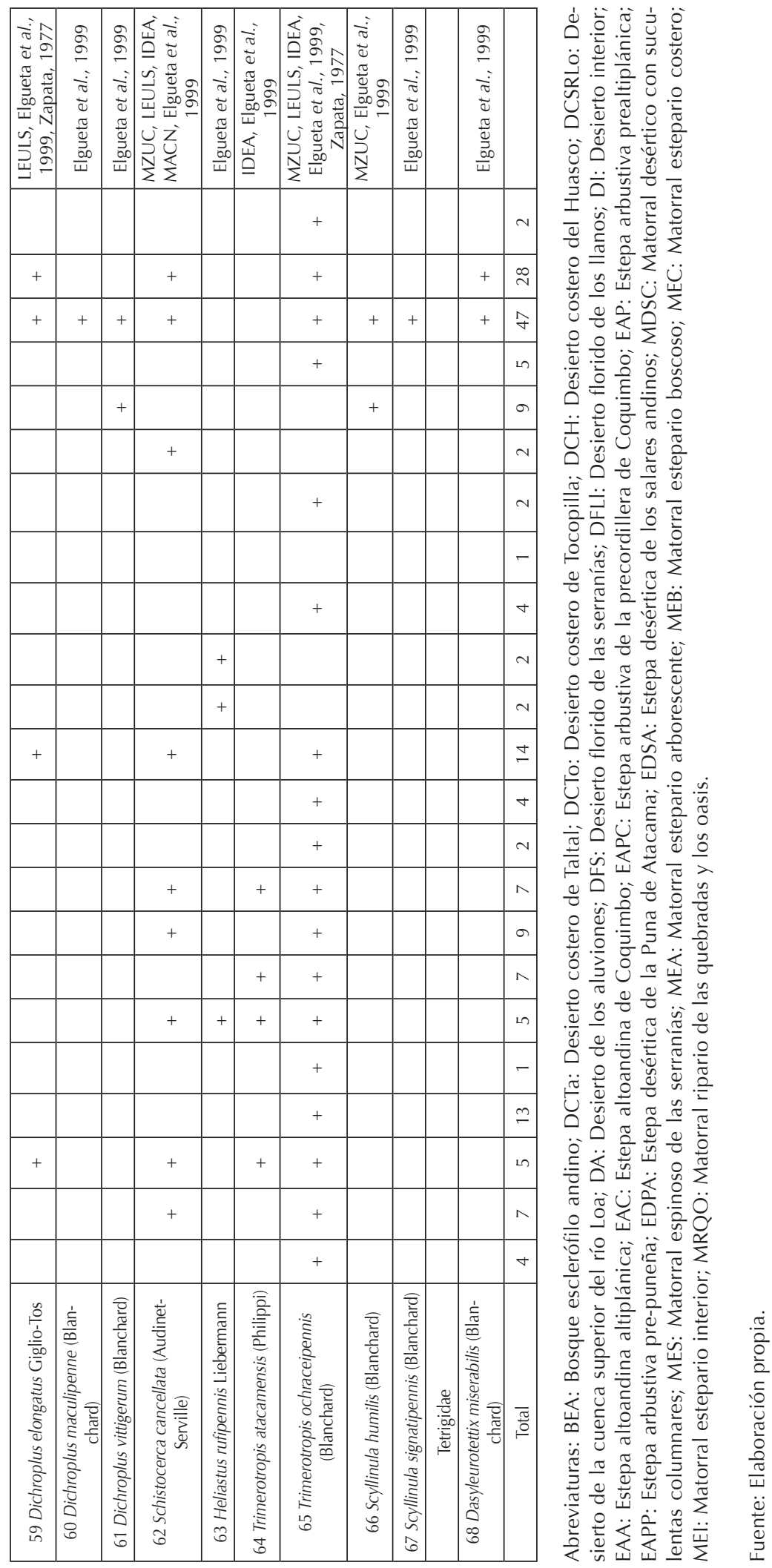


Figura $\mathrm{N}^{\circ} 1$

Riqueza específica de Orthoptera en relación a formaciones vegetales descritas en las provincias biogeográficas de Atacama y Coquimbo $\left(18^{\circ}-32^{\circ}\right.$ LS)

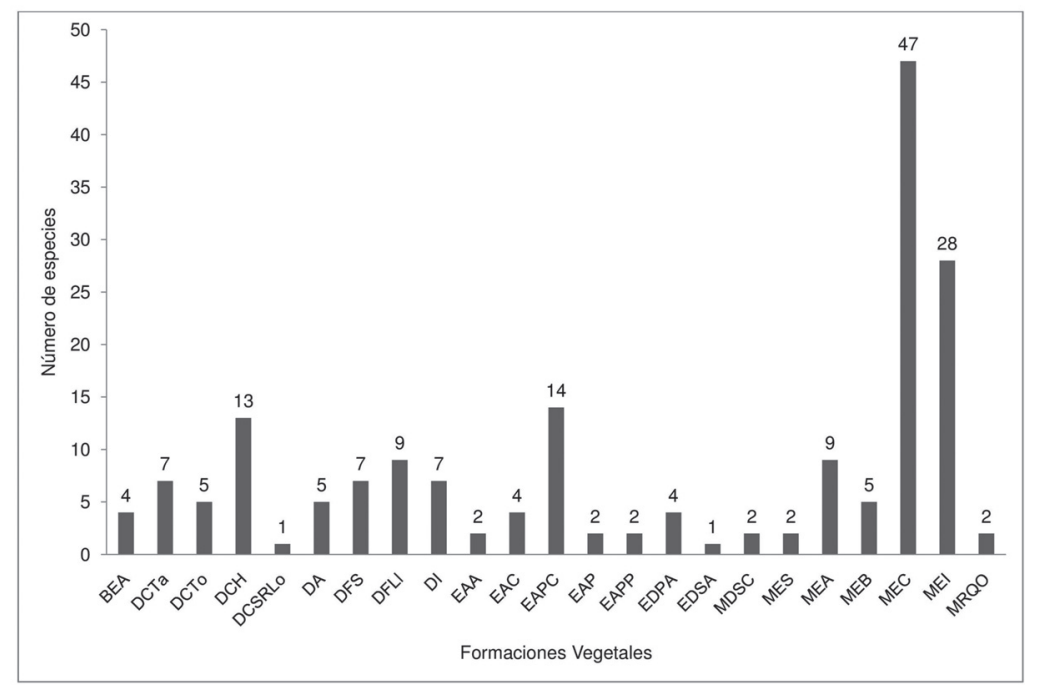

Abreviaturas: BEA: Bosque esclerófilo andino; DCTa: Desierto costero de Taltal; DCTo: Desierto costero de Tocopilla; DCH: Desierto costero del Huasco; DCSRLo: Desierto de la cuenca superior del río Loa; DA: Desierto de los aluviones; DFS: Desierto florido de las serranías; DFLI: Desierto florido de los Ilanos; DI: Desierto interior; EAA: Estepa altoandina altiplánica; EAC: Estepa altoandina de Coquimbo; EAPC: Estepa arbustiva de la precordillera de Coquimbo; EAP: Estepa arbustiva prealtiplánica; EAPP: Estepa arbustiva pre-puneña; EDPA: Estepa desértica de la Puna de Atacama; EDSA: Estepa desértica de los salares andinos; MDSC: Matorral desértico con suculentas columnares; MES: Matorral espinoso de las serranías; MEA: Matorral estepario arborescente; MEB: Matorral estepario boscoso; MEC: Matorral estepario costero; MEI: Matorral estepario interior; MRQO: Matorral ripario de las quebradas y los oasis.

Fuente: Elaboración propia.

\section{Distribución geográfica de Orthoptera}

La mayoría de los registros se concentraron en ambientes costeros y valles interiores entre $\operatorname{los} 27^{\circ}$ y $32^{\circ} \mathrm{S}$, franja mayoritariamente ubicada en la provincia biogeográfica de Coquimbo $\left(28^{\circ}-32^{\circ} \mathrm{S}\right.$, subregión Chilena Central). Gran parte de la franja latitudinal entre los $21^{\circ}$ y los $26^{\circ}$, correspondiente a zonas del desierto interior, valles transversales y precordillera, presentaron escasos registros (Figuras $N^{\circ} 2$ y $\mathrm{N}^{\circ} 3$ ).

La familia Acrididae presentó la mayor cantidad de registros dentro de la zona bajo estudio, los cuales en su mayoría se ubicaron entre los $28^{\circ}$ y $32^{\circ}$ de latitud sur. Los registros de las especies de esta familia indican una distribución desde los $18^{\circ} 4^{\prime}$ hasta los $32^{\circ} \mathrm{S}$. Sin embargo, esta familia presenta una amplia distribución en la zona central de Chile y la Patagonia, abarcando las provincias biogeográficas de Santiago (subregión Chilena Central), Islas Juan Fernández, Maule, Bosque Valdiviano y Bosque MagaIlánico (subregión Subantártica), las cuales comprenden la franja latitudinal entre los $32^{\circ}$ y $47^{\circ} \mathrm{S}$ y pertenecen a la región Andina (Figuras $\mathrm{N}^{\circ} 2$ y $\mathrm{N}^{\circ} 3$ ).

La familia Rhaphidophoridae, representada en este estudio solo por una especie, presenta una distribución desde la provincia biogeográfica de Coquimbo $\left(30^{\circ} \mathrm{S}\right)$ hasta la provincia del Páramo Magallánico $\left(48^{\circ} \mathrm{S}\right.$, subregión Subantártica en la región Andina).

Tettigoniidae se encuentra distribuida entre las provincias de Atacama (zona de transición Sudamericana) y del Bosque Valdiviano 


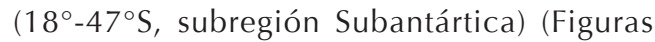
$\mathrm{N}^{\circ} 2$ y $\left.\mathrm{N}^{\circ} 3\right)$.

Gryllidae presenta escasos registros en el extremo norte de la provincia de Atacama; estos se concentran en la provincia de Coquimbo $\left(30^{\circ} \mathrm{S}\right)$ y se extienden hasta los $47^{\circ} \mathrm{S}$ (Figura $\mathrm{N}^{\circ} 3$ ).

La familia Proscopiidae presenta una amplia distribución dentro de la zona de estudio. Sin embargo, los registros se concentran en la provincia de Coquimbo. Esta familia se distribuye hasta la provincia del Bosque Magallánico (subregión Subantártica) (Figuras $\mathrm{N}^{\circ}$ 2 y $\left.N^{\circ} 3\right)$.

La familia Tristiridae presenta registros desde los $23^{\circ} 13^{\prime} \mathrm{S}$, los cuales se concentran mayoritariamente en los $30^{\circ} \mathrm{S}$. Algunas especies de este grupo (i.e. Atacamacris diminuta Carbonell \& Mesa, Enodisomacris curtipennis
Cigliano, Uretacris lilai Liebermann) presentan solo un registro distribucional dentro de la zona bajo estudio, encontrándose su distribución bastante restringida dentro de la provincia de Atacama. En Chile, las especies de esta familia presentan una distribución hasta la provincia del Páramo Magallánico (Figura $\mathrm{N}^{\circ} 2$ ).

La familia Ommexechidae se distribuye en gran parte de las provincias de Atacama y Coquimbo desde los $18^{\circ} 31^{\prime} \mathrm{S}$. Algunas especies de este grupo (i.e. Conometopus brevirostrum Ronderos, Conometopus penai Ronderos) presentan escasos registros, los cuales se restringen a la provincia de Atacama (Figura $N^{\circ} 3$ ).

Las familias Romaleidae y Tetrigidae estuvieron representadas solo por un género y una especie. Su distribución en Chile abarca desde la provincia de Coquimbo hasta la provin-

Figura $\mathrm{N}^{\circ} 2$

Distribución espacial de las principales familias de Orthoptera (Insecta), presentes en las provincias biogeográficas de Atacama y Coquimbo

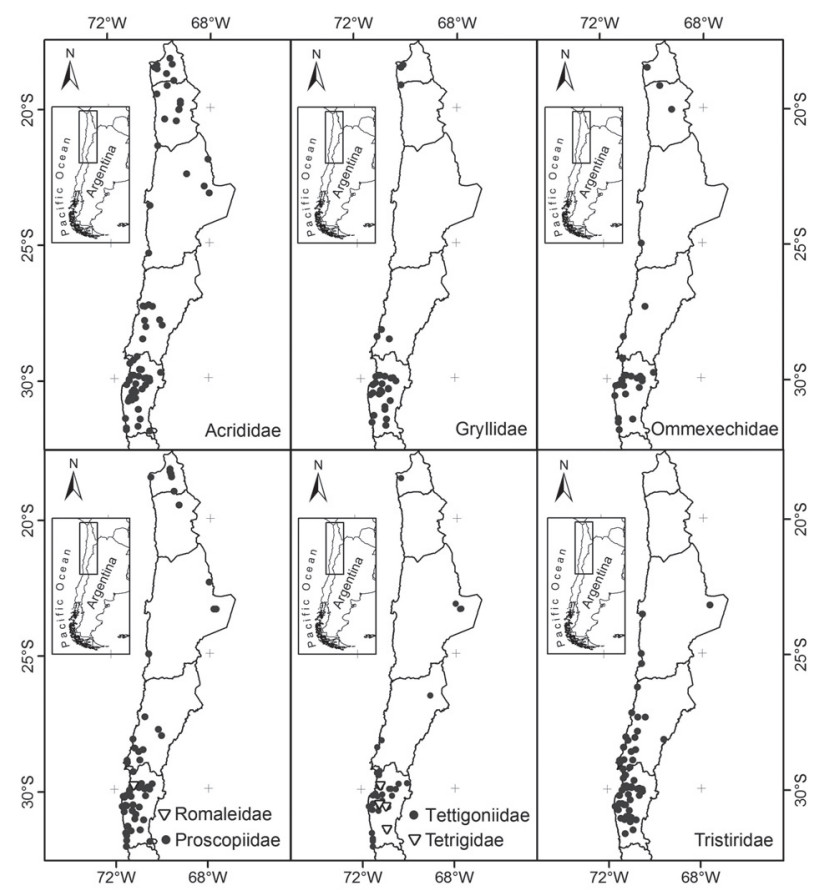

Fuente: Elaboración propia. 
Figura $\mathrm{N}^{\circ} 3$

Distribución espacial de los principales géneros de Orthoptera (Insecta), presentes en las provincias biogeográficas de Atacama y Coquimbo

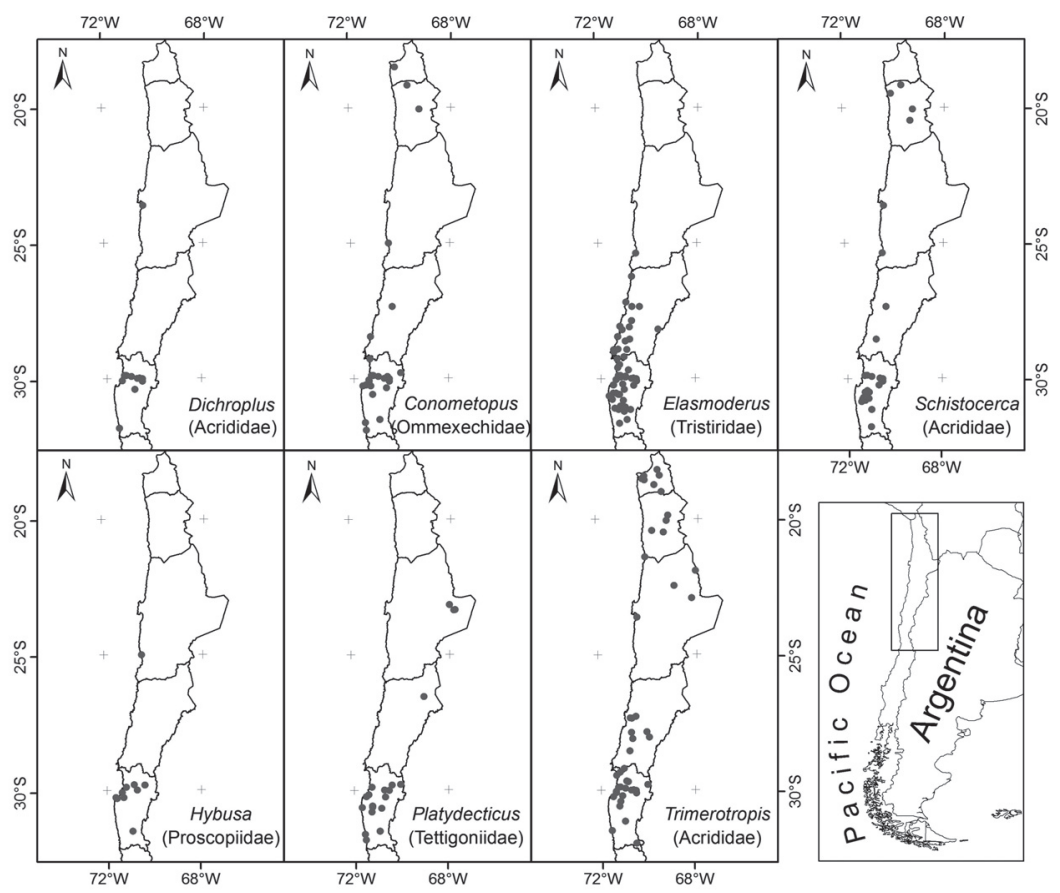

Fuente: Elaboración propia.

cia del Bosque Valdiviano (Romaleidae) y la provincia del Maule (Tetrigidae) (Figura $N^{\circ} 2$ ).

\section{Consideraciones finales}

Las familias más diversas dentro de las provincias biogeográficas de Atacama y Coquimbo fueron Tettigoniidae (24 especies y 12 géneros), seguida de Proscopiidae (14 especies y 6 géneros). Cabe destacar que ambas familias son los grupos más diversos en Chile. Por otra parte, la mayor diversidad específica observada en la formación vegetal Matorral estepario costero concuerda con lo registrado por Pizarro-Araya y Jerez (2004) para Gyriosomus (Coleoptera: Tenebrionidae), género de gran diversificación y que pertenece a la tribu Nycteliini, grupo endémico de ambientes áridos y semiáridos de Sudamérica austral (Flores, 2000). Estos resultados podrían indicar la existencia de zonas de "hotspots" para la diversidad de Orthoptera en esta zona mediterránea, considerando que pueden ser definidas como zonas con altos niveles de endemismos y concentración de especies (Myers et al., 2000).

Los sectores con mayor presencia de especies correspondieron a ambientes costeros entre $\operatorname{los} 27^{\circ}$ y $32^{\circ}$ de latitud sur, los cuales se encuentran ubicados en su mayoría dentro de la provincia biogeográfica de Coquimbo. Sin embargo, la mayor riqueza específica se podría relacionar con una mayor cantidad de registros asociados a sectores de fácil acceso. De acuerdo a Jerez (2000), lo anterior puede indicar que la mayoría de la información disponible en colecciones y en la literatura corresponde a recolecciones efectuadas cerca de poblados o de la red vial, lo que en conjunto no aporta información completa del área.

De acuerdo a O'Brien (1971) la entomofauna de la provincia de Atacama (zona de transición Sudamericana) se encontraría re- 
lacionada con la existente en la provincia de Coquimbo (subregión Chilena Central de la región Andina). Nuestros resultados muestran que gran parte de los géneros y familias de Orthoptera presentan una distribución continua a lo largo de la franja ocupada por ambas provincias biogeográficas, extendiéndose también dentro de la subregión Subantártica.

Para la zona Norte y Centro-norte de Chile la existencia de trazos generalizados en ciertas especies de plantas (i.e. Apocynaceae), ha permitido corroborar la relación entre la región Andina y la zona de transición Sudamericana (Hechem et al., 2011). Estas relaciones se han basado también en la existencia de especies de insectos (e.g. Orthoptera: Tristiridae; Coleoptera: Carabidae, Curculionidae, Pselaphidae y Tenebrionidae; Diptera: Simulidae; Hymenoptera: Formicidae, Pompilidae y Vespidae; y Lepidoptera: Noctuidae) comunes a ambas zonas (Morrone, 2001; Morrone, 2006).

Análisis panbiogeográficos realizados para la zona de transición Sudamericana (provincia de Atacama) y la región Andina (provincia de Coquimbo), indican que los elementos de la entomofauna presente (e.g., Orthoptera, Coleoptera, Diptera, Hymenoptera y Lepidoptera) están relacionados, entre ambas provincias; en este sentido diversos autores (Kuschel, 1969; Morrone, 1994; Morrone, 2006) establecen que la mayor parte de la biota de la región Andina originalmente proveniente de la Patagonia se extendió gradualmente hacia el norte dentro de la zona de transición Sudamericana con la aparición de comunidades áridas, antecedentes que concuerdan con lo documentado por Domínguez et al. (2006), Roig-Juñent et al. (2006) y Hechem et al. (2011).

Uno de los elementos endémicos de América del Sur corresponde a la familia Tristiridae, la cual representa un componente biótico erémico representativo de zonas áridas (Kuschel, 1964; Roig-Juñent y Flores, 2001; Donato, 2006). Esta familia presenta una gran distribución en la región andino-patagónica desde el paralelo $10^{\circ}$ de latitud sur hasta Tierra del Fuego, y extendiéndose hacia la costa chilena (Cabrera y Willink, 1973; Cigliano, 1989a; Cigliano, 1989b; Cigliano, 1991; Cigliano et al., 1989; Donato, 2006).
Este taxón muestra un origen a partir de las regiones de la Puna y Patagonia, presentando líneas de dispersión hacia las regiones de desierto del Norte Chico (Donato, 2006). Uno de los grupos representativos de esta familia lo constituye el género Elasmoderus Saussure, el cual presenta una distribución restringida a la franja comprendida entre $\operatorname{los} 26^{\circ}$ y $32^{\circ}$ de latitud sur (Cigliano et al., 1989), región que corresponde a las provincias de Atacama y Coquimbo. De acuerdo a Donato (2006) este género junto a los géneros monoespecíficos Uretacris Liebermann y Enodisiomacris Cigliano, se habrían originado en las zonas desérticas de Sudamérica a partir de efectos vicariantes que dividieron la zona de la Patagonia a finales del mioceno.

La insuficiente información sobre la distribución de ortópteros queda demostrada por la falta de prospecciones en gran parte del área de estudio. Además se debe considerar la existencia de escasos trabajos sobre aspectos taxonómicos, ecológicos y biogeográficos de este grupo para las zonas áridas y semiáridas de Chile. En este sentido, se espera que el presente estudio constituya una primera aproximación al conocimiento de la diversidad de Orthoptera de las provincias biogeográficas septentrionales de Chile.

Se espera además que los antecedentes distribucionales entregados aquí representen un elemento relevante para la elaboración de un inventario general que permita la posterior delimitación de áreas diversas y endémicas. Esto a su vez permitiría la identificación de zonas prioritarias en la conservación de la biota del norte de Chile.

\section{Referencias bibliográficas}

ALCAYAGA, O.E.; PIZARRO-ARAYA, J.; ALFARO, F.M. \& CEPEDA-PIZARRO, J. Arañas (Arachnida: Araneae) asociadas a agroecosistemas en el Valle de Elqui (Región de Coquimbo, Chile). Revista Colombiana de Entomología, 2013, Vol. 39, No 1, p. 150154.

ALFARO, F.M.; PIZARRO-ARAYA, J. \& FLORES, G.E. Epigean tenebrionids (Coleoptera: Tenebrionidae) from the Choros Archipe- 
lago (Coquimbo Región, Chile). Entomological News, 2009, Vol. 120, N², p. 125-130.

ALFARO, F.M.; PIZARRO-ARAYA, J.; BODINI, A. y CEPEDA-PIZARRO, J. Composición y distribución del ensamble de Orthoptera (Insecta) en una cuenca árida del norte-centro de Chile. Revista de la Facultad de Ciencias Agrarias, 2011, Vol. 43, № 1, p. 97-110.

ARTIGAS, J.N. Entomología económica. Insectos de interés agrícola, forestal, médico y veterinario (nativos, introducidos y susceptibles de ser introducidos). Vol. 1. Concepción: Ediciones Universidad de Concepción, 1994.

CABRERA, A.L. y WILLINK, A. Biogeografía de América Latina. Washington, D.C.: OEA. Serie Biología, Monografía No 13, 1973.

CARBONELL, C.S. y MESA, A. Dos nuevos géneros y especies de acridoideos andinos (Orthoptera). Revista Peruana de Entomología, 1972, Vol. 15, p. 95-102.

CEPEDA-PIZARRO, J.; PIZARRO-ARAYA, J. y VÁSQUEZ, H. Variación en la abundancia de Arthropoda en un transecto latitudinal del desierto costero transicional de Chile, con énfasis en los tenebriónidos epígeos. Revista Chilena de Historia Natural, 2005a, Vol. 78, p. 651-663.

CEPEDA-PIZARRO, J.; PIZARRO-ARAYA, J. y VÁSQUEZ, H. Composición y abundancia de artrópodos epígeos del Parque Nacional Llanos de Challe: impactos del ENOS de 1997 y efectos del hábitat pedológico. Revista Chilena de Historia Natural, 2005b, Vol. 78, p. 635-650.

CEPEDA-PIZARRO, J.; VEGA, S.; VÁSQUEZ, H. y ELGUETA, M. Morfometría y dimorfismo sexual de Elasmoderus wagenknechti (Liebermann) (Orthoptera: Tristiridae) en dos eventos de irrupción poblacional. Revista Chilena de Historia Natural, 2003, Vol. 76, p. 417-435.

CEPEDA-PIZARRO, J.; VEGA, S.; ELGUETA, M. y PIZARRO-ARAYA, J. Algunos antecedentes meteorológicos que explican las irrupciones poblacionales de Elasmoderus wagenknechti (Liebermann) (Orthoptera: Tris- tiridae) en la región del semiárido de Chile. IDESIA (Chile), 2006, Vol. 24, p. 49-64.

CEPEDA-PIZARRO, J.; VEGA, S.; VÁSQUEZ, H.; ELGUETA, M. \& PIZARROARAYA, J. Demography of two populations outbreaks of Elasmoderus wagenknechti (Orthoptera: Tristiridae) in the semiarid region of Chile. Neotropical Entomology, 2007, Vol. 36, p. 495-502.

CIGLIANO, M.M. Revisión sistemática de la familia Tristiridae (Insecta: Orthoptera). Boletín de la Sociedad de Biología de Concepción (Chile), 1989a, Vol. 60, p. 89-91.

CIGLIANO, M.M. A cladistic analysis of the family Tristiridae (Orthoptera, Acridoidea). Cladistics, 1989b, Vol. 5, p. 379-393.

CIGLIANO, M.M. Congruencia taxonómica en la familia Tristiridae (Orthoptera, Acridoidea). Revista de la Sociedad Entomológica Argentina, 1991, Vol. 49, № 1-4, p. 85-101.

CIGLIANO, M.M.; RONDEROS, R.A. \& KEMP, W.P. Revision of the genus Elasmoderus Saussure (Orthoptera: Tristiridae). The Canadian Entomologist, 1989, Vol. 121, p. 225-243.

COWLING, R.M.; RUNDEL, P.W.; LAMONT, B.B.; ARROYO, M.K. \& ARIANOUTSOU, M. Plant diversity in Mediterranean-climate regions. Trends in Ecology and Evolution, 1996, Vol. 11, p. 362-366.

DONATO, M. Historical biogeography of the family Tristiridae (Orthoptera: Acridomorpha) applying dispersal-vicariance analysis. Journal of Arid Environments, 2006, Vol. 66, p. 421-434.

DOMÍNGUEZ, M.C.; ROIG-JUNENT, S.; TASSIN, J.J.; OCAMPO, F.C. \& FLORES, G.E. Areas of endemism of the Patagonian steppe: an approach based on insect distributional patterns using endemicity analysis. Journal of Biogeography, 2006, Vol. 33, p. 1527-1537.

EADES, D.C.; OTTE, D.; CIGLIANO, M. M. \& BRAUN, H. Orthoptera Species File. 2013. Versión 5.0. Disponible en internet: http://orthoptera.speciesfile.org 
ELGUETA, M.; CAMMOUSEIGHT, A. Y CARBONNEL, C.S. Catálogo de Orthoptera (Insecta) de Chile. Publicación Ocasional, Museo Nacional de Historia Natural (Chile), 1999, Vol. 54, p. 1-60.

ELGUETA, M. y BARRÍA, C. Registro en altura de Enodisomacris curtipennis Cigliano, 1989 (Orthoptera: Tristiridae), en la zona costera sur de la región de Antofagasta. Boletín del Museo Nacional de Historia Natural, Chile, 2008, Vol. 57, p. 133-138.

FLORES, G.E. Cladistic analysis of the Neotropical tribe Nycteliini (Coleoptera: Tenebrionidae). Journal of the New York Entomological Society, 2000, Vol. 108, p. 13-25.

FLORES, G.E. \& PIZARRO-ARAYA, J. Revision of species of the genus Praocis Eschscholtz, 1829 (Coleoptera: Tenebrionidae). Part 1: Subgenus Praocis s. str. Zootaxa, 2012, Vol. 3336, p. 1-35.

GAJARDO, R. La Vegetación Natural de Chile: clasificación y distribución geográfica. Santiago de Chile: Editorial Universitaria, 1994.

GASTON, K.J. Global patterns in biodiversity. Nature, 2000, Vol. 405, p. 220-227.

GRISMADO, C.J. Description of Birabenella, a new genus of goblin spiders from Argentina and Chile (Araneae: Oonopidae). American Museum Novitates, 2010 , Vol. 3693, p. 1-21.

HECHEM, V.; ACHERITOBEHERE, L. Y MORRONE, J.J. Patrones de distribución de las especies de Cynanchum, Diplolepis y Tweedia (Apocynaceae: Asclepiadoideae) de América del Sur austral. Revista de Geografía Norte Grande, 2011, Vol. 48, p. 45-60.

JEREZ, V. Diversidad y patrones de distribución geográfica de insectos coleópteros en ecosistemas desérticos de la región de Antofagasta, Chile. Revista Chilena de Historia Natural, 2000, Vol. 73, p. 79-92.

KOÇAK, A. \& KEMAL, M. Replacement names among the genus and family groups taxa in Orthoptera. Miscellaneous Papers
Centre for Entomological Studies Ankara, 2008, Vol. 141, p. 1-8.

KUSCHEL, G. Problems concerning an austral region. In: GRESSIT, J.L.; LINDROTH, C.H.; FORSBERG, F.R.; FLEMING, A. \& TURBOTT, E.G. (editors). Pacific basin biogeography. Honolulu: Bishop Museum Press, 1964, p. 443-449.

KUSCHEL, G. Biogeography and ecology of South American Coleoptera. In: FITKAU, E. J.; ILLIES, J.; KLINGE, H.; SCHWABE, G. H. \& SIOLI, H. (editors). Biogeography and ecology in South America. La Haya: Junk, 1969, p. 709-722.

LUCAS, S.M. \& INDICATTI, R.P. Description of two new species of Lycinus (Araneae: Nemesiidae). Zoologia, 2010, Vol. 27, No 3, p. 425-430.

MELLO-LEITÃO, C. DE. Estudio monográfico de los Proscópidos. Revista del Museo de La Plata (N.S.), 1939, Vol. 1, p. 279-448.

MORONI, B.J. Irrupción de Elasmoderus rabiosus (Liebermann) en el Norte Chico. Noticiario Mensual, Museo Nacional de Historia Natural (Chile), 1972, Vol. 192, p. 3-7.

MORRONE, J.J. Distributional patterns of species of Rhytirrhinini (Coleoptera: Curculionidae) and the historical relationships of the Andean provinces. Global Ecology and Biogeography Letters, 1994, № 4, p. 188-194.

MORRONE, J.J. Delimitation of the Central Chilean Subregion and its provinces, based mainly on arthropod taxa. Biogeographica, 2000, Vol. 76, №3, p. 97-106.

MORRONE, J.J. Biogeografía de América Latina y el Caribe. Zaragoza: M\&T-Manuales \& Tesis SEA, Sociedad Entomológica Aragonesa, 2001.

MORRONE, J.J. Presentación sintética de un nuevo esquema biogeográfico de América Latina y el Caribe. En: COSTA, C.; VANIN, S.; LOBO, J. y MELIC, A. (editores). Proyecto de Red Iberoamérica de Biogeografía y Entomología Sistemática PrIBES 2002. Zaragoza: Monografías Tercer Milenio, Sociedad Ento- 
mológica Aragonesa (SEA), 2002, Vol. 2, p. 267-275.

MORRONE, J.J. La zona de transición Sudamericana: caracterización y relevancia evolutiva. Acta Entomológica Chilena, 2004a, Vol. 28, No 1, p. 41-50.

MORRONE, J.J. Panbiogeografía, componentes bióticos y zonas de transición. Revista Brasileira de Entomologia, 2004b, Vol. 48, Nº 2, p.149-162.

MORRONE, J.J. Biogeographic areas and transition zones of Latin America and the Caribbean Islands based on panbiogeographic and cladistic analyses of the entomofauna. Annual Review of Entomology, 2006, Vol. 51, p. 467-94.

MYERS, N.; MITTERMEIER, R.A.; MITTERMEIER, C.G.; DA FONSECA, G.A.B., \& KENT, J. Biodiversity hotspots for conservation priorities, Nature, 2000, Vol. 403, p. 853-858.

NICKLE, D.A. New species of the genus Anisophya Karabag from Chile (Orthoptera: Tettigoniidae: Phaneropterinae). Journal of Orthoptera Research, 2011, Vol. 20, № 2, p. 163-172.

O'BRIEN, C.W. The biogeography of Chile through entomofaunal regions. Entomological News, 1971, Vol. 82, p. 197-207.

OJANGUREN-AFFILASTRO, A.A. Nuevos aportes al conocimiento del género Brachistosternus en Chile, con la descripción de dos nuevas especies (Scorpiones, Bothriuridae). Boletín de la Sociedad de Biología de Concepción (Chile), 2002, Vol. 73, p. 37-46.

OJANGUREN-AFFILASTRO, A.A. Un nuevo Brachistosternus Pocock 1893 del norte de Chile (Scorpiones, Bothriuridae). Revista Ibérica de Aracnología, 2004, Vol. 10, p. 69-74.

OJANGUREN-AFFILASTRO, A.A. Notes on the genus Brachistosternus (Scorpiones, Bothriuridae) in Chile, with the description of two new species. Journal of Arachnology, 2005, Vol. 33, p. 175-192.

OJANGUREN-AFFILASTRO, A.A. \& MATTONI, C. I. A new species of Brachistosternus from Chilean central Andes (Scorpiones: Bothriuridae). Studies on Neotropical Fauna and Environment, 2006, Vol. 41, p. 79-85.

OJANGUREN-AFFILASTRO, A.A.; AGUSTO, P.; PIZARRO-ARAYA, J. \& MATTONI, C. I. Two new scorpion species of genus Brachistosternus (Scorpiones: Bothriuridae) from northern Chile. Zootaxa, 2007, Vol. 1623, p. 55-68.

OJANGUREN-AFFILASTRO, A.A.; MATTONI, C.I.; OCHOA, J.A \& PRENDINI, L. Rumikiru, n. gen. (Scorpiones: Bothriuridae), a New Scorpion Genus from the Atacama Desert. American Museum Novitates, 2012, Vol. 3731, p. 1-43.

PIZARRO-ARAYA, J. y JEREZ, V. Distribución geográfica del género Gyriosomus Guérin-Méneville, 1834 (Coleoptera: Tenebrionidae): una aproximación biogeográfica. Revista Chilena de Historia Natural, 2004, Vol. 77, p. 491-500

PIZARRO-ARAYA, J. \& FLORES, G.E. Two new species of Gyriosomus Guérin-Méneville from Chilean coastal desert (Coleoptera: Tenebrionidae: Nycteliini). Journal of the New York Entomological Society, 2004, Vol. 112, p. 121-126.

PIZARRO-ARAYA, J.; CEPEDA-PIZARRO, J. y FLORES, G.E. Diversidad taxonómica de los artrópodos epígeos de la Región de Atacama (Chile): estado del conocimiento. En: SQUEO, F.A.; ARANCIO, G. y GUTIÉRREZ, J.R. (editores). Libro Rojo de la Flora Nativa y de los Sitios Prioritarios para su Conservación: Región de Atacama. La Serena: Ediciones Universidad de La Serena, 2008, p. 257-274.

PIZARRO-ARAYA, J.; CEPEDA-PIZARRO, J.; BARRIGA, J.E. \& BODINI, A. Biological vulnerabilities analysis, with an emphasis on economically important arthropods in the Elqui Valley (Coquimbo Region, Chile). Ciencia e Investigación Agraria, 2009, Vol. 36, p. 215-228.

PIZARRO-ARAYA, J.; ALFARO, F.M.; CASTILLO, J.P.; OJANGUREN-AFFILASTRO, A.A.; AGUSTO, P. \& CEPEDA-PIZARRO, J. Assemblage of arthropods in the Quebrada del Morel private protected area (Atacama Region, 
Chile). The Pan-Pacific Entomologist, 2012a, Vol. 88, p. 1-14.

PIZARRO-ARAYA, J.; VERGARA, O.E. y FLORES, G.E. Gyriosomus granulipennis Pizarro-Araya \& Flores 2004 (Coleoptera: Tenebrionidae) un caso extremo a conservar. Revista Chilena de Historia Natural, 2012b, Vol. 85, p. 345-349.

ROIG-JUÑENT, S. y FLORES, G.E. Historia biogeográfica de las áreas áridas de América del Sur Austral. En: LLORENTE BUSQUETS, J. y MORRONE, J.J. (editores). Introducción a la biogeografía en Latinoamérica: teorías, conceptos, métodos y aplicaciones. México D.F.: Las prensas de Ciencias, Facultad de Ciencias, UNAM, 2001, p. 257-266.

ROIG-JUÑENT, S.; DOMÍNGUEZ, M.C.; FLORES, G.E. \& MATTONI, C. Biogeographic history of South American arid lands: A view from its arthropods using TASS analysis. Journal of Arid Environments, 2006, Vol. 66, p. 404-420.

RONDEROS, R.A. Un nuevo Conometopini del norte de Chile (Orthoptera, Acrididae, Ommexechinae). Neotropica, 1972, Vol. 18, $N^{\circ} 55$, p. 31-36.

RONDEROS, R.A. Notas para una revisión de la subfamilia Ommexechinae. III. Una nueva especie de la tribu Conometopini (Orthoptera, Acrididae). Revista de la Sociedad Entomológica Argentina, 1973, Vol. 34, p. 25-29.

SQUEO, F.A.; CAVIERES, L.A.; ARANCIO, G.; NOVOA, J.E.; MATTHEI, O.; MARTICORENA, C.; RODRÍGUEZ, R.; ARROYO, M.T. K. y MUÑOZ, M. Biodiversidad de la flora vascular en la Región de Antofagasta, Chile. Revista Chilena de Historia Natural, 1998, Vol. 71, p. 571-591.

SQUEO, F.A.; ARANCIO, G.; MARTICORENA, C.; MUÑOZ, M. y GUTIÉRREZ, J.R. Diversidad vegetal en la IV Región de Coquimbo, Chile. En: SQUEO, F.A.; ARANCIO,
G. y GUTIÉRREZ, J.R. (editores). Libro rojo de la flora nativa y de los sitios prioritarios para su conservación: Región de Coquimbo. La Serena: Ediciones Universidad de La Serena, 2001, p. 149-158.

SQUEO, F.A.; ARANCIO, G.; LETELIER, L.; MARTICORENA, A.; MUÑOZ-SCHICK, M.; LEÓN-LOBOS, P. y ARROYO, M.T.K. Estado de conservación de la Flora nativa de la región de Atacama. En: SQUEO, F.A.; ARANCIO, G. y GUTIÉRREZ, J.R. (editores). Libro Rojo de la Flora Nativa y de los Sitios Prioritarios para su Conservación: Región de Atacama. La Serena: Ediciones Universidad de La Serena, 2008, p. 45-59.

VALDIVIA, D.E.; PIZARRO-ARAYA, J.; CEPEDA-PIZARRO, J. Y OJANGUREN-AFFILASTRO, A.A. Diversidad taxonómica y densoactividad de solífugos (Arachnida: Solifugae) asociados a un ecosistema desértico costero del centro norte de Chile. Revista de la Sociedad Entomológica Argentina, 2008, Vol. 67, №1-2, p. 1-10.

VALDIVIA, D.E.; PIZARRO-ARAYA, J.; BRIONES, R.; OJANGUREN-AFFILASTRO, A.A. \& CEPEDA-PIZARRO, J. Taxonomical diversity and abundance of solpugids (Arachnida: Solifugae) in coastal ecotopes of northcentral chile. Revista Mexicana de Biodiversidad, 2011, Vol. 82, p. 1234-1242.

VERA, A. Orden Orthoptera (grillos, langostas y saltamontes). En: SABALL, P.; ARROYO, M.T.K.; CASTILLA, J.C.; ESTADES, C.; LADRÓN DE GUEVARA, J.M.; LARRAÍN, S.; MORENO, C.; RIVAS, F.; ROVIRA, J.; SÁNCHEZ, A. y SIERRALTA, L. (editores). Biodiversidad de Chile. Patrimonio y desafíos. Santiago de Chile: Comisión Nacional del Medio Ambiente, 2006, p. 141-144.

ZAPATA, C.S. Notas sobre biología, distribución y comportamiento alimenticio en cuatro especies de acrídidos chilenos. Revista de la Sociedad Entomológica Argentina, 1977, Vol. 36, No 1-4, p. 147-151. 